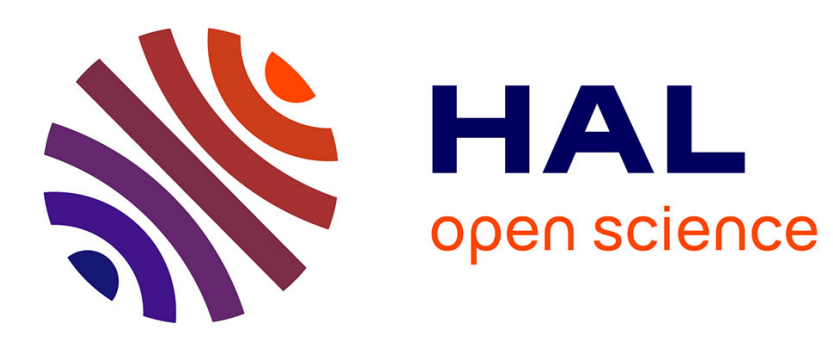

\title{
Chronique inachevée d'une réflexion collective sur le document
}

\author{
Jean-Michel Salaün
}

\section{To cite this version:}

Jean-Michel Salaün. Chronique inachevée d'une réflexion collective sur le document. Communication \& langages, 2004, 140. sic_00001028

\section{HAL Id: sic_00001028 \\ https://archivesic.ccsd.cnrs.fr/sic_00001028}

Submitted on 12 Jul 2004

HAL is a multi-disciplinary open access archive for the deposit and dissemination of scientific research documents, whether they are published or not. The documents may come from teaching and research institutions in France or abroad, or from public or private research centers.
L'archive ouverte pluridisciplinaire HAL, est destinée au dépôt et à la diffusion de documents scientifiques de niveau recherche, publiés ou non, émanant des établissements d'enseignement et de recherche français ou étrangers, des laboratoires publics ou privés. 
Jean-Michel Salaün

Professeur

Enssib

17,21 Bd du 11 novembre 1918

69623 Villeurbanne Cedex

salaun@enssib.fr

\section{Chronique inachevée d'une réflexion collective sur le document}

Jean-Michel Salaün

Version du 17-02-2004

Communication et Langages $\mathrm{n}^{\circ} 140$ Juin 2004

\section{Chapô :}

Cet article présente un travail collectif de réflexion sur le document dans sa bascule vers le numérique mené au sein d'un réseau de chercheurs du département Sciences et technologies de l'information de la communication du CNRS. La rédaction collective d'un texte, menée notamment grâce à un dispositif d'annotations, a conduit à un inventaire analytique des recherches en cours. Celui-ci comprend trois entrées : la forme, le texte et le medium qui font ressortir les traits saillants de l'évolution en cours et les questions encore en suspens. En particulier, la construction de plus en plus courante de documents à la volée transforme radicalement leur stabilité traditionnelle. L'avancement de la réflexion collective pourrait déboucher sur l'élaboration d'une théorie du document.

\section{Abstract :}

This paper presents a collective analytic work on the document in its transition to digital form carried out within a network of researchers of the CNRS Information and Communication Science and Technology (STIC) Department. The collective drafting of a text, carried out with a device of annotations, built an analytical inventory of research in progress. It stressed three topics: the form, the text and the medium which reflect the radical transformations that are taking place and the issues still going on. In particular, the more and more dynamic construction of documents actually transforms their traditional stability. The advance of the collective thought could lead to the development of a theory of the document.

\section{Texte :}

Curieusement, le « document», omniprésent dans notre quotidien, n'a été que peu soumis à la réflexion critique des chercheurs. Alors qu'une section entière sur " information, communication et connaissance " figure en bonne place dans le projet d'établissement du CNRS, le terme « document » n'y apparaît pas comme si ce n'était ni une notion pertinente, ni un objet susceptible d'analyse. Du côté de l'université, si les sciences de l'information en ont débattu au moment de leur fondation en France, la discussion a vite tourné court, le terme d'« information » prenant le dessus, un peu comme si le contenu prévalait sur le contenant. Le même mouvement est sensible dans les appellations, savoir-faire et formations professionnels où l'on passe de bibliothécaire à documentaliste puis à « spécialiste de l'information » décliné 
enfin sous des vocables divers, suivant ainsi le mouvement anglo-saxon où " Information science » a remplacé « Librarianship ».

Sans doute, l'objet-document est trop familier, trivial, la notion est trop intuitive. Ils n'étaient ou ne paraissaient pas problématiques, dans tous les sens du terme. Pourtant aujourd'hui le numérique bouscule profondément le document, devenu électronique. Et les chercheurs français sont nombreux à prendre le document numérique comme objet de recherche, directement ou indirectement. Mais ils sont dispersés géographiquement, éclatés dans leurs approches et ils n'échangent régulièrement qu'entre tenants d'une spécialité pointue.

L'absence d'une définition commune explique peut-être cet éclatement, mais fait problème pour des chercheurs dont c'est souvent le terrain principal d'investigation. Peser lucidement les décisions technologiques, leurs effets et mesurer les conséquences des transformations, se révèle hasardeux faute d'avoir au préalable vraiment cerné les contours de l'objet de son travail. Pire, même si les uns et les autres le déplorent souvent, les relations entre les tenants des sciences de l'ingénieur et ceux des sciences humaines et sociales sont quasi-inexistantes, à la notable exception près de la linguistique, ou parfois difficiles et conflictuelles, les premiers reléguant les sciences sociales à l'« applicatif», les seconds considérant les sciences de l'ingénieur comme du domaine de la " technique », chacun de ces qualificatifs renvoyant à un domaine exotique, étranger, mal connu voire hostile. Même si, bien entendu, je force le trait et que l'on trouve, des deux côtés, de plus en plus de chercheurs et même des écoles de pensée qui ne tombent pas dans ce travers, il reste suffisamment répandu pour laisser craindre que bien des chercheurs en informatique jouent aux apprentis sorciers, appréhendant de façon trop lacunaire les conséquences sociales des multiples choix algorithmiques, pendant que bien des chercheurs en sciences sociales continuent de poser a posteriori une critique sur les outils trop distanciée, méconnaissant trop leur construction et fonctionnement, pour être vraiment entendue ou simplement pertinente.

Dans ce contexte de dispersion et de méconnaissance, il était prioritaire de se mettre d'accord sinon sur une définition commune du terme "document » au moins sur un repérage de ses différentes dimensions, une cartographie des recherches en cours et une énumération des problèmes émergeants. Et l'organisation en réseau pluridisciplinaire s'est avérée d'une efficacité certaine ${ }^{1}$.

\section{Les modalités d'une réflexion collective}

Pour engager cette réflexion entre des chercheurs qui souvent ne se connaissaient pas ou mal, nous avons donc utilisé pleinement les potentialités de la structure en réseau et celles des échanges électroniques. L'objectif, clairement exprimé dès le départ du travail, quelques mois après la fondation du réseau, était la rédaction d'un texte collectif sur le sujet. L'intérêt de l'opération était double, conceptuel et social. D'une part, compte tenu de la diversité des points de vue, il paraissait difficile, voire impossible, à un seul auteur d'appréhender

\footnotetext{
${ }^{1}$ Le département Sciences et technologies de l'information et de la communication du CNRS a mis en place dans le courant de l'année 2002 une série de réseaux thématiques pluridisciplinaires fondés sur l'hypothèse que, une nouvelle donne se jouant dans le numérique par la prise en compte du symbolique par les ingénieurs, elle nécessitait de nouvelles analyses basées sur la rencontre entre les disciplines et sans doute aussi de nouvelles méthodes d'investigation. Ainsi a été fondé le réseau « Documents et contenu : création, indexation, navigation » (en abrégé : RTP-DOC). Dans cet article, nous ne rendrons compte que de l'une des actions transversales du réseau : la réflexion collective sur la notion de document, renvoyant le lecteur intéressé au site du réseau pour la présentation du reste de son activité http://rtp-doc.enssib.fr.
} 
lucidement la globalité des problématiques. D'autre part, la dynamique collective incitait les chercheurs à lire les contributions diverses et prendre en compte la pluralité et, sinon fondait une communauté de recherche, au moins établissait une connaissance mutuelle et la base commune qui faisait défaut jusqu'à présent. Enfin un troisième bénéfice est apparu en cours d'opération : la publicité faite autour de la démarche a largement contribué à la notoriété du réseau et ainsi, par un effet classique de résonance, a alimenté la dynamique collective.

Voici, brièvement résumées, les différentes étapes du processus de rédaction, tel qu'il s'est réalisé au jour où nous écrivons ces lignes sachant qu'il est encore en cours et que nous ne pouvons dire quand (ou même si) il s'achèvera :

- une première phase préliminaire de contributions et discussions libres, soit par la liste générale du réseau, soit dans ses instances de pilotage, s'est conclue par un séminaire qui a réuni une trentaine de chercheurs. Le résultat de cette première phase a été un balisage des approches et recherches en cours qui a montré la pertinence d'un découpage en trois entrées (intitulées à ce moment du processus : forme, signe, relation). Sur la base de tous les matériaux recueillis, j'ai synthétisé un premier texte qui a été signé d'un pseudonyme : Roger T. Pédauque (pour RTP-DOC). S'agissant d'un document de travail reprenant de nombreuses contributions de natures diverses, il était difficile de lui attribuer une responsabilité auctoriale précise. Ce choix, au départ pragmatique et amusé, s'est avéré d'une réelle efficacité. Il a en effet rendu caduque toute présomption de propriété intellectuelle sur tel ou tel argument du texte et a donné d'emblée une consistance symbolique à la dynamique en l'incarnant dans l'épopée d'un personnage fictif.

- La véritable rédaction collective s'est développée dans la deuxième phase. Le texte du susdit Roger a été mis en ligne sur un site d'archives ouvertes ${ }^{2}$. Chaque paragraphe était numéroté et il était indiqué que le lecteur pouvait participer à sa critique et donc à son amélioration en envoyant ses remarques à l'adresse de l'auteur, précisant le numéro du ou des paragraphes incriminés. Parallèlement, une liste de diffusion dédiée était créée pour recueillir et partager les différents apports. Les membres du comité de pilotage du réseau étaient inscrits à cette liste ainsi que, au fur et à mesure, les différents intervenants sur le texte. Une large publicité sur l'opération a été faite à la fois à l'intérieur du réseau et dans diverses listes spécialisées ou même individuellement auprès d'experts du domaine. La discussion a été ouverte pendant deux mois. Elle a rencontré un vrai succès puisque le texte a été téléchargé plus de mille fois et qu'une trentaine de chercheurs ou professionnels du document ont proposé des corrections, amendements ou commentaires. Ceux-ci étaient intégrés au fur et à mesure dans une version électronique du texte sous forme d'annotations ${ }^{3}$. La conclusion de cette phase a été, évidemment, une nouvelle version, enrichie et corrigée, du texte qui fut assez simple à reconstruire compte tenu du dispositif choisi. Cette seconde version a été mise en ligne début juillet 2003 sur le site d'archives et est encore, au moment où nous écrivons ces lignes, la version de référence ${ }^{4}$. Nous disposons donc dorénavant d'un texte dressant un panorama large des recherches en

\footnotetext{
${ }^{2}$ Document : forme, signe et relation, les re-formulations du numérique Roger T. Pédauque. Article. 28 avril 2003. Working paper. http://archivesic.ccsd.cnrs.fr/sic_00000413.html

${ }^{3}$ L'ensemble des contributions intermédiaires et le détail de la démarche est consultable sur le site du RTP à la rubrique « Roger T. Pédauque ».

${ }^{4}$ Document : forme, signe et médium, les re-formulations du numérique Roger T. Pédauque. Article. 08 juillet 2003. Working paper. http://archivesic.ccsd.cnrs.fr/documents/archives0/00/00/05/11/index fr.html
} 
cours, des problématiques, des avancées et des questions encore non résolues. Plus encore sans doute que la précédente version, celle-ci a rencontré un très large succès en terme de téléchargements, tout comme d'ailleurs sa traduction anglaise disponible depuis septembre 2003.

- La troisième, et dernière à ce jour, étape est donc de retrouver le processus scientifique traditionnel pour tirer un véritable profit de recherche de la dynamique lancée. A cette fin, le texte de Roger $\mathrm{T}$. Pédauque est pris comme point de départ à des appels à contribution $^{5}$, des dossiers de revues, des débats électroniques ou réels, mais cette fois les articles sont signés par leurs auteurs, révisés par les pairs, et les responsabilités éditoriales sont assumées. Le point d'orgue de l'opération sera la semaine du document numérique qui se tiendra du 21 au 25 juin 2004 à La Rochelle ${ }^{6}$. Au moment où j'écris ces lignes, cette étape est en cours et il n'est pas possible d'en anticiper les résultats.

Il m'est difficile de mesurer lucidement l'intérêt et les biais de la méthode employée d'écriture collective. Je manque du recul indispensable. Je dirais qu'elle a vraisemblablement plus favorisé l'accumulation, l'affinement que le débat ou la contestation. Les propositions ou affirmations ont plus été remplacées, amendées que débattues ou contestées. Ainsi l'intelligence collective s'est manifestée par son encyclopédisme et sa rigueur plutôt que par son originalité ou par le débat d'idées et la cohérence d'un raisonnement. De par son mode d'écriture le texte a donc au moins deux vertus et une limite. Il rend compte de la diversité des travaux de recherche et, au-delà de celle-ci, il montre l'importance d'une appréhension globale de l'objet en pointant des convergences ou des contradictions entre des approches et des résultats. Mais sa limite est de se présenter plus comme un inventaire que comme une analyse réellement scientifique. Il balise un champ de recherche mais n'en construit pas les fondements théoriques.

Compte tenu du constat fait au début de cet article, je crois pouvoir dire que le premier objectif de mise en commun et de construction d'une connivence suffisante pour engager un dialogue interdisciplinaire a été atteint. Mais le succès même de l'opération nous engage alors à aller plus loin.

\section{Une première lecture}

Chaque paragraphe du texte pédauquien a été relu et critiqué et contient des incitations à la réflexion, néanmoins je crois que l'on peut déjà en tirer aussi quelques leçons générales.

Il est possible ainsi de repérer des étapes, ici schématisées, dans l'histoire récente de la numérisation des documents. Le document traditionnel repose sur un support, un texte et une légitimité. Une première phase de numérisation a fait ressortir ses structures internes, l'importance des métadonnées pour son traitement et la complexité de sa validation. Une seconde phase, sans doute commencée mais dont l'aboutissement vers un "Web sémantique ${ }^{7}$ » reste incertain, insiste sur le format $\mathrm{XML}^{8}$, qui intègre la structure mais la

\footnotetext{
${ }_{6}^{5}$ Pour la revue $\mathrm{I} 3<\mathrm{http}: / /$ www.revue-i3.org $>$

${ }^{6} \mathrm{http}: / / \mathrm{sdn} 2004 . u n i v-1 \mathrm{r} . \mathrm{fr} /$

${ }^{7}$ Le Web sémantique est une proposition de Tim Berners-Lee. C'est un Web dont le contenu peut être appréhendé et exploité par des machines. Ainsi, le Web sémantique devrait fournir des services plus précis et complexes aux internautes que le Web actuel (répondre directement à une question, sélectionner, localiser et activer le service nécessaire, articuler des besoins complémentaires et complexes, etc.). C'est aussi une infrastructure complétant le contenu informel du Web actuel avec de la connaissance formalisée. Pour une présentation complète :
} 
détache de la mise en forme par les feuilles de style, voudrait s'appuyer sur des « ontologies ${ }^{9}$ " pour retrouver et reconstruire les textes, et met en avant l'accès personnalisé. Il y a dans cette évolution générale un sens dont il faudrait mieux comprendre l'orientation, les conséquences et les limites.

Par ailleurs, le découpage en trois entrées a été affiné entre la première et la seconde version du texte. Il permet une cartographie des recherches en cours sur le document numérique. Celle-ci mériterait d'ailleurs d'être plus structurée et pourrait devenir un outil de repérage et éventuellement de pilotage des recherches.

Dans la première entrée sur le signe ou la forme, trois communautés de chercheurs se croisent sans toujours bien se connaître : les traiteurs d'image, les éditeurs numériques et la communauté du Web. Même s'ils partent de problématiques différentes, ils convergent nettement aujourd'hui dans la compréhension des structures logiques du document et dans les interrogations sur les formes perceptibles. Le travail principal concerne les formats, au sens informatique, c'est à dire des outils de mise en forme. D'une certaine façon, le numérique a déplacé la question du support du document, qui en assurait la stabilité en fixant l'inscription, vers la problématique de sa structure. L'inscription transformée en signal se déplace d'un support à l'autre et la stabilité du document réside alors dans celle de l'organisation du signal. La popularisation de la norme XML marque une étape mais elle laisse encore largement ouverte la problématique de la perception. En effet, en séparant de façon radicale la structure logique d'un texte de sa représentation visuelle, elle autorise des traitements formels différents pour un même contenu à une échelle inédite.

La seconde entrée sur le texte (il faut comprendre ce terme dans un sens générique sans référence à un mode d'expression particulier, comme synonyme de contenu) fait se rencontrer les chercheurs en linguistique, en sciences de l'information et en ingénierie des connaissances. Le problème principal est de construire des modèles permettant de traiter le contenu des documents, afin de les retrouver, et éventuellement les réagencer pour en produire de nouveaux, adaptés à la demande du lecteur. Il s'agit de produire du sens pour le lecteur en dépassant la confusion de l'accumulation des informations, à partir notamment d'une modélisation de données sur les documents, les métadonnées. Au-delà de la performance des outils, la question posée est celle de la construction des savoirs dans la relation entre les métalangages et les documents eux-mêmes. Manipuler des ontologies, c'est à dire entre autres articuler des concepts, n'est pas sans effet sur l'organisation de nos connaissances, particulière à une communauté ou générique pour une société.

La troisième entrée sur le médium comprend deux terrains sensiblement différents : d'une part la communication organisationnelle, d'autre part les médias de masse. On y trouve principalement des chercheurs en télécommunication ; c'est ici aussi que les sciences sociales et les sciences de la communication sont majoritaires. Le document y fonde son statut dans

Rapport de l'Action spécifique Web sémantique pilotée par Jean Charlet, Philippe Laublet et Chantal Reynaud accessible à l'Url : http://rtp-doc.enssib.fr/archiveas.html

${ }^{8}$ eXtended Markup Language. Langage de balises qui sépare le contenu, la structure et la présentation d'un document. La souplesse de ce format autorise notamment une très grande modularité des parties de document et ainsi permet de combiner différents éléments avec souplesse chacun gardant sa propre logique. Pour une présentation simple, conférence de Vincent Quint Le Web de demain, 25-10-2001 accessible à l'Url : http://isdn.enssib.fr/archives/transversal/JDN/webdemain/webdemain.html

${ }^{9}$ Une ontologie en ingéniérie des connaissances est constituée de l'ensemble des concepts d'un domaine hiérarchiquement organisés et éventuellement reliés par des relations de calcul. Pour une présentation claire et détaillée, cf. chapitre correspondant du rapport de l'AS Web sémantique, op. cit. 
une diffusion dépassant l'intime et l'éphémère, en s'articulant avec d'autres dans des collections. C'est à dire qu'un document n'existe que dans une collectivité élargie et une durée pour alimenter un patrimoine de connaissances partagées. Deux pratiques sociales très « normées » se modifient au travers du Web. D'une part, la frontière entre la communication inter-personnelle et la communication «flottante» ou publique, entre correspondance privée et publicité, se déplace et tous les codes sociaux et modalités organisationnelles qui s'y rapportent sont alors ébranlés. D'autre part, illustrées par le préfixe " hyper- » (hypertexte, hypermédia), les relations entre documents se distribuent différemment entre partage et liens, entre mise en ligne et mise en réseau. Suite à ces deux déplacements, la notion de publication, qui s'était stabilisée au cours des siècles, se transforme brutalement et celle de bibliothèque, dont les contours étaient limités par la taille des collections et l'implantation physique et institutionnelle, explose en bibliothèque numérique, centralisée ou distribuée et largement accessible.

Le tout peut être résumé en un tableau :

\section{Recherches sur le document numérique}

\begin{tabular}{|l|l|l|l|}
\hline & \multicolumn{1}{|c|}{ Chercheurs } & Objet et résultats & $\begin{array}{l}\text { Aboutissement et } \\
\text { interrogation }\end{array}$ \\
\hline Forme & $\begin{array}{l}\text { Traiteurs d'image, édition } \\
\text { numérique, W3C }\end{array}$ & $\begin{array}{l}\text { Support/Structure } \\
\text { Formats }\end{array}$ & $\begin{array}{l}\text { XML } \\
\text { Perception? }\end{array}$ \\
\hline Texte & $\begin{array}{l}\text { Linguistique, Sciences de } \\
\text { l'information, ingénierie } \\
\text { des connaissance }\end{array}$ & $\begin{array}{l}\text { Sens/Meta } \\
\text { Moteurs, ontologies }\end{array}$ & $\begin{array}{l}\text { Web sémantique } \\
\text { Savoirs? }\end{array}$ \\
\hline Medium & $\begin{array}{l}\text { Télécom, Sciences de la } \\
\text { communicaton, gestion, } \\
\text { Sciences sociales }\end{array}$ & $\begin{array}{l}\text { Collection/hyper } \\
\text { Portails, collaboratifs }\end{array}$ & $\begin{array}{l}\text { Bibliothèque numérique } \\
\text { Publication? }\end{array}$ \\
\hline
\end{tabular}

Si l'on croise ces entrées, on constate qu'une évolution possible, explorée par bien des chercheurs en pointe sur ce champ mais non certaine, serait que les documents structurés, informés et contextualisés, rejoignent des bases de données, centralisées ou distribuées, et que l'ensemble des fichiers s'apparente de plus en plus à un ou plusieurs vastes jeux où des briques de différentes tailles, formes et usages seraient agencées selon des configurations très variées. Selon cette hypothèse dont la validité est sans doute variable selon la spécificité des contextes, un document n'aurait alors d'existence à proprement parler qu'à deux moments : éventuellement celui de sa conception par son auteur qui devra le visualiser ou l'entendre, pour s'assurer qu'il correspond à ses choix et surtout celui de sa re-construction par un lecteur.

Nous percevons très clairement les prémisses de cette évolution, par exemple dans la multiplication de sites Web dynamiques, où les pages sont construites à la volée à chaque requête de l'internaute, ou encore dans la page actualité de Google réactualisée toute les quinze minutes par un moteur balayant 500 sites d'informations journalistiques ${ }^{11}$. On assiste même à des sortes de mise en abîme où des lecteurs mettent en ligne leurs impressions dans des chroniques souvent reliées entre elles, les blogs.

\footnotetext{
${ }^{10}$ World Wide Web Consortium. Organisme de recommandation technique visant à conduire le Web à son plein potentiel. Pour une bonne présentation du W3C : conférence de Vincent Quint Le Web de demain, op.cit.

${ }^{11} \mathrm{http}: / /$ news.google.fr/
} 
Nous entrevoyons aussi bien des problèmes qu'elle soulève, par exemple, celui de la validation et de la hiérarchisation ou celui de la responsabilité éditoriale ; il est beaucoup plus aventureux d'en prévoir les avancées, les résistances ou déviances et donc les conséquences, sinon pour dire qu'elles seront à coup sûr importantes et durables.

\section{Vers une théorie du document}

Pour mieux cerner la notion de document en général dont le numérique est à la fois un révélateur et un facteur d'évolution, il est donc éclairant de regrouper les recherches en cours selon les trois entrées citées. Mais ce classement pourrait bien aller au-delà du simple repérage auquel il nous a servi jusqu'ici. Mon hypothèse est qu'il pourrait permettre de poser les bases d'une théorie du document. Ainsi, d'une façon un peu ironique, la notion de document, dont la définition ne semblait plus un enjeu pour la recherche, trouverait peut être les moyens de se construire au moment même où son objet paraît échapper.

Dans la continuité de la démarche engagée, l'élaboration d'une telle théorie ne saurait être que collective. Elle engage en effet trop de savoirs spécialisés pour pouvoir être le produit d'un seul individu. Ce sera donc peut être un nouvel enjeu d'écriture pour Roger T. Pédauque..

En attendant, remarquons qu'une définition générale est déjà esquissée dans la conclusion du texte pédauquien : Un document ne serait finalement qu'une convention entre des hommes qui fonderait une part de leur humanité, de leur capacité à vivre ensemble et dont les modalités anthropologiques (lisibilité-perception, signe), intellectuelles (intelligibilitéassimilation, texte) et sociales (sociabilité-intégration, médium) devraient non seulement être efficientes prises chacune séparément, mais encore être cohérentes entre elles. La première partie de la définition, la convention, relève principalement des sciences sociales tandis que la seconde, les trois modalités, concerne plus directement les sciences de l'ingénieur.

Concernant ces dernières, la proposition peut être alors, me semble-t-il, résumée sur un nouveau tableau :

\section{Comment se construit un document?}

\begin{tabular}{|l|l|l|l|}
\hline & \multicolumn{1}{|c|}{ Définition } & \multicolumn{1}{c|}{ Caractéristique } & \multicolumn{1}{c|}{ Identité } \\
\hline Perception & Une inscription repérable & Identifiable, lisible & $\begin{array}{l}\text { au delà du silence et du } \\
\text { bruit }\end{array}$ \\
\hline Intellect & Un texte construit & Compréhensible, interprétable & $\begin{array}{l}\text { au delà du sensible et du } \\
\text { confus }\end{array}$ \\
\hline Social & Une référence partagée & $\begin{array}{l}\text { Appropriable, crédible, } \\
\text { critiquable }\end{array}$ & $\begin{array}{l}\text { au delà de l'intime et de } \\
\text { l'éphémère }\end{array}$ \\
\hline
\end{tabular}

Les trois lignes marquent la correspondance humaine des trois entrées repérées sur l'objetdocument : Perception et Forme ; Intellect et Texte ; Social et Médium. Les trois colonnes précisent la nature d'un document en le décrivant, le qualifiant et l'identifiant. Le tableau ne fait pas la différence entre document traditionnel et document numérique. Il n'est présenté ici qu'à titre de proposition, de suggestion, pour amorcer un débat qui doit maintenant s'ouvrir.

Insistons sur le fait que les trois entrées représentent toujours trois pôles et non trois domaines étanches. Ainsi les problèmes de perception, par exemple, ne peuvent être traités indépendamment des questions intellectuelles ou sociales, sauf à prétendre que la perception d'un document relève de l'inné ! Néanmoins, poser la question documentaire à partir de la perception revient à mettre un accent plus marqué sur les problématiques de supports, de formats, de structures etc. et suppose de développer des analyses et des outils capables de rendre compte finement de leurs logiques et dimensions. La proposition théorique vise donc à ne pas privilégier une approche par rapport à une autre, tout en admettant la nécessité de tenir 
deux impératifs qui peuvent être concurrents dans l'organisation concrètes des travaux : le développement individuel de chacune des approches et leur superposition et croisement. Ce programme ne peut se concevoir que collectivement et dans l'interdisciplinarité.

Reste la première partie de la proposition de définition. Peut-être parce que les chercheurs en sciences sociales ont été moins représentés dans la rédaction collective, la question de la convention, en fait celle de la fonction sociale du document, y est peu discutée et mériterait de plus amples réflexions et développements. A vrai dire, le terme de " convention » n'est pas même pas employé dans le texte d'origine, c'est moi qui l'ajoute ici. Il me semble, en effet, plus approprié que celui, trop précis, de « contrat de lecture » qui y est mis en avant. En fait s'il y a bien accord pour autoriser une lecture et la construction d'un sens, il s'agit plutôt d'un accord tacite, flou, d'une connivence sociale, même s'il se concrétise souvent dans des règles précises.

Je formulerais volontiers l'hypothèse qu'il se construit sous nos yeux un nouveau compromis entre une multiplicité d'acteurs pour ré-inventer des documents ou des artefacts de substitution. Dans ce processus, le numérique joue un rôle majeur mais il n'est sûrement pas le seul phénomène en cause. En l'absence des outils pour une analyse pertinente le jeu des acteurs et les multiples micro-négociations se réalisent "à l'aveugle », privilégiant les ajustements de court terme et les intérêts immédiats. Le risque est le manque de cohérence et les malentendus multiples. Ainsi en est-il, pour ne prendre que deux exemples, des discours et initiatives contradictoires et confus sur le droit d'auteur dans le numérique ou encore des multiples et envahissants parasitages de la publication numérique.

Dans cette perspective, le numérique en venant bousculer les modalités de construction des documents ne serait qu'un vecteur de multiplication, de renouvellement et peut-être un des ferments de la transformation des conventions qui les ont institués. 